\title{
The role of pancreatic insulin secretion in neonatal glucoregulation. I. Healthy term and preterm infants
}

\author{
J M Hawdon, A Aynsley-Green, K G M M Alberti, M P Ward Platt
}

\begin{abstract}
The glucoregulatory role of insulin in adult subjects is undisputed. However, less is known about the secretion of insulin and its actions in the neonatal period, either for healthy subjects, or for those at risk of disordered blood glucose homoeostasis. The relationships between blood glucose and plasma immunoreactive insulin concentrations were therefore examined in 52 healthy children (aged 1 month-10 years), 67 appropriate birth weight for gestational age (AGA) term infants, and 39 AGA preterm neonates.

In children and AGA neonates, plasma immunoreactive insulin concentration was positively related to blood glucose concentration. However, although both groups of neonates had significantly lower blood glucose concentrations than children, plasma immunoreactive insulin concentrations were significantly higher in both term and preterm neonates, when compared with children. The variation in plasma immunoreactive insulin concentrations was greater for neonates than for children.
\end{abstract}

These data suggest, that compared with older subjects, plasma immunoreactive insulin concentrations are high in newborn babies and that neonatal pancreatic insulin secretion is less closely linked to circulating blood glucose concentrations. There are important implications for the interpretation of studies in hypoglycaemic and hyperglycaemic neonates.

\section{(Arch Dis Child 1993;68:274-279)}

Studies of healthy adults have demonstrated that insulin is secreted by the pancreas, primarily in response to circulating blood glucose concentrations with cessation of secretion when blood glucose falls below $3 \mathrm{mmol} / 1 .{ }^{1-3}$ In turn, insulin inhibits hepatic glucose production and the release of fatty acids from adipose tissue. ${ }^{45}$ In adults, glucose appears to be the most important regulator of pancreatic endocrine function, and circulating plasma insulin concentrations closely control blood glucose concentrations through effects on glucose production and peripheral consumption.

The relationship between insulin and glucose metabolism in younger subjects is less clear. It is known that there are differences between adult and neonatal subjects in terms of pancreatic structure and patterns of insulin secretion. ${ }^{6-8}$ Therefore, differences in gluco- regulation by insulin might be expected. However, the neonatal pancreatic endocrine response to varying levels of glycaemia has not been described in detail in healthy infants, and the relative importance of insulin and other glucoregulatory hormones is disputed. ${ }^{910}$

Unfortunately, many studies of glucoregulation in the newborn period have described only hypoglycaemic and hyperglycaemic infants, with no reference to data from normal infants. In addition, these studies have not considered the hormonal regulation of the provision of alternative fuels such as ketone bodies or the effect the presence of these fuels may have on pancreatic insulin secretion. Studies that have given 'normal ranges' for concentrations of glucose and insulin have not examined the interrelationships of glucose, other intermediary metabolites, and insulin. ${ }^{11}$

We have already described the patterns of metabolic adaptation in appropriate birth weight for gestational age (AGA) term and preterm infants, and have demonstrated that circulating fuel concentrations, and the interrelationships between them, vary with gestational and postnatal age. ${ }^{12}$ However, it is not known whether changes in glucoregulatory hormone concentrations cause these variations.

We have also described the hormonal and metabolic associations of neonatal hypoglycaemia and hyperglycaemia and have shown that, for these infants, plasma insulin concentrations were not related to blood glucose concentrations, nor to glucose production rates. ${ }^{13}$ Previous studies of small for gestational age infants have suggested that hyperinsulinism contributes to hypoglycaemia. ${ }^{14-18}$ However, these studies are difficult to interpret in the absence of data regarding the relationship between insulin and glucose in healthy AGA infants. Therefore, we have investigated the relationship between plasma insulin concentrations and blood concentrations of glucose and intermediary metabolites in healthy, term and preterm AGA infants.

\section{Subjects and methods}

The subjects of the present study were those AGA infants, described in a recent cross sectional study, ${ }^{12}$ for whom sufficient plasma sample volumes $(100 \mu \mathrm{l})$ were available to measure plasma insulin concentrations. Cord blood samples were available from some infants and postnatal samples were collected from babies aged 0-6 days. All babies were clinically stable. Samples were collected before 
a feed, and at least 2 hours after the previous feed, by heelprick from a warmed foot. An aliquot of $40 \mu \mathrm{l}$ whole blood were added to $200 \mu 13 \%$ perchloric acid, and $250 \mu \mathrm{l}$ blood were collected into a heparinised container. Samples were stored on ice before separation and freezing. Blood concentrations of glucose and ketone bodies and plasma non-esterified fatty acid concentrations were determined by microenzymatic methods, as described previously. ${ }^{12}$

Prefeed plasma insulin concentrations were measured for 39 AGA preterm infants and 67 AGA term infants. The data from these AGA infants were compared with the blood glucose and intermediary metabolite concentrations and plasma insulin concentrations of a group of 52 healthy children, aged 1 month to 10 years, who were the subjects of a study examining metabolic responses to surgery and were sampled, after an overnight fast, immediately before surgery. ${ }^{19}$

Plasma insulin concentrations were measured by double antibody radioimmunoassay, which had an assay sensitivity of $1 \mathrm{mU} / \mathrm{l}$, an intra-assay coefficient of variation of $6 \%$, and interassay coefficients of variation of $7.5 \%$ and $6.8 \%$ at $7.0 \mathrm{mU} / 1$ and $44.2 \mathrm{mU} / \mathrm{l}$, respectively. The antibody used in the assay cross reacts fully with proinsulin and split proinsulins. Therefore these molecules contributed to the measured immunoreactive insulin concentration.

Plasma insulin, blood ketone body, and non-esterified fatty acid (NEFA) concentrations had log normal distributions, and the lowest limits of detection for glucose, ketone body, and insulin concentrations were 1.5 $\mathrm{mmol} / \mathrm{l}, 0.02 \mathrm{mmol} / \mathrm{l}$, and $0.1 \mathrm{mmol} / 1$, respectively. Therefore, non-parametric statistical analysis was employed, using the SPSS-X package. Spearman correlation was used to describe the relationships between variables. The Mann-Whitney U test was used to compare groups of subjects. A multiple regression analysis model was constructed to investigate the correlations between certain variables, while controlling for the effects of other variables. $\log _{10}$ values were used for insulin, ketone body, and NEFA concentrations in this analysis. The insulin:glucose ratio was calculated for each infant as a means of correcting the insulin concentrations for the circulating blood glucose concentrations. A birthweight SD score was calculated for each infant. ${ }^{12}$

Details of infants studied. Results are range (median)

\begin{tabular}{|c|c|c|c|}
\hline & $A G A$ preterm & $A G A$ term & Children \\
\hline Gestation (weeks) & $26-36(32)$ & $37-41(39)$ & \\
\hline Birth weight (g) & $830-2925(1800)$ & $2575-4565(3290)$ & \\
\hline Birthweight SD score & -1.0 to $2.0(0.0)$ & -1.0 to $3.8(0.3)$ & \\
\hline Age (hours) & $1-139(82)$ & $1-140(51)$ & \\
\hline Age (months) & & & $1-129(25)$ \\
\hline \multicolumn{4}{|l|}{ Cord blood: } \\
\hline Glucose $(\mathrm{mmol} / \mathrm{l})$ & $2 \cdot 4-5 \cdot 7(4 \cdot 3)$ & $2 \cdot 6-5 \cdot 8(3.5)$ & \\
\hline NEFA $(\mathrm{mmol} / \mathrm{l})$ & $0.03-0.25(0.10)$ & $0.04-0.53(0.09)$ & \\
\hline Ketone body $(\mathrm{mmol} / \mathrm{l})$ & $0.03-0.60(0.17)$ & $0.04-1.04(0.24)$ & \\
\hline Insulin (mU/l) & $<1.0-22.7(2.5)$ & $2 \cdot 0-29 \cdot 3(6 \cdot 4)$ & \\
\hline \multicolumn{4}{|l|}{ Postnatal: } \\
\hline Glucose $(\mathrm{mmol} / \mathrm{l})$ & $1 \cdot 5-12 \cdot 2(4 \cdot 1)$ & $1 \cdot 8-5 \cdot 4(3 \cdot 7)$ & $3 \cdot 5-7 \cdot 7(4 \cdot 9)$ \\
\hline NEFA $(\mathrm{mmol} / \mathrm{l})$ & $0.01-1.04(0.05)$ & $0.04-1.34(0.36)$ & $0 \cdot 13-2 \cdot 38(0 \cdot 72)$ \\
\hline Ketone body $(\mathrm{mmol} / \mathrm{l})$ & $0.01-0.51(0.03)$ & $0.01-2.06(0.20)$ & $0.02-4.16(0.31)$ \\
\hline Insulin $(\mathrm{mU} / \mathrm{l})$ & $<1.0-69.8(3.0)$ & $<1.0-28.5(1.5)$ & $<1.0-7.5(1.0)$ \\
\hline
\end{tabular}

\section{Results}

The clinical details of the infants, the blood glucose and intermediary metabolite concentrations, and the plasma immunoreactive insulin concentrations are shown in the table. There were no significant differences between term and preterm infants in cord
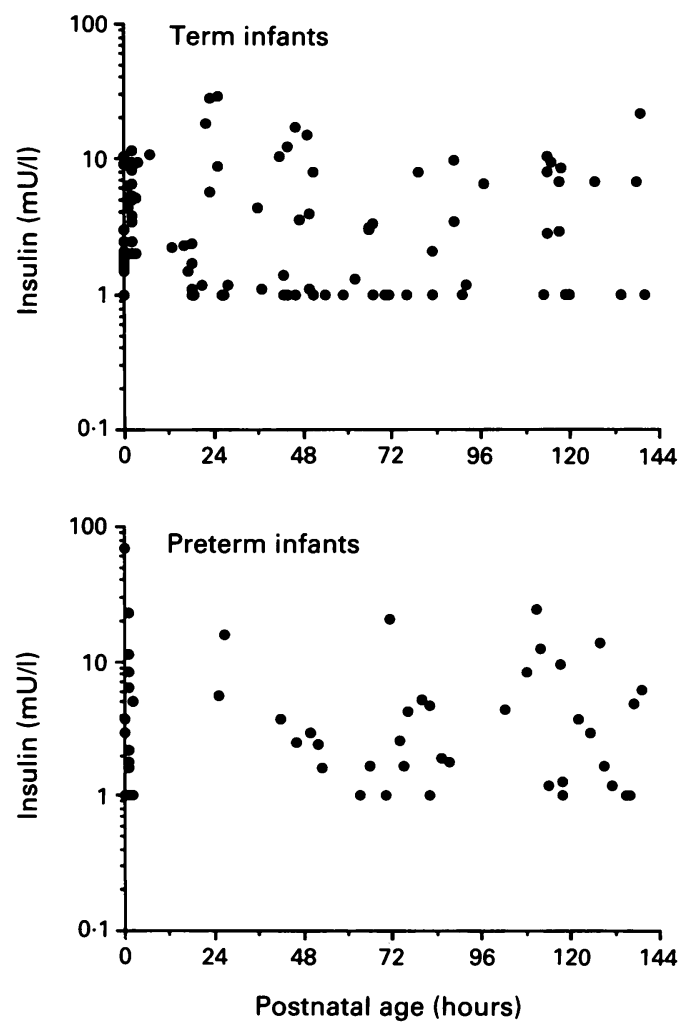

Figure 1 Relationship of plasma insulin concentration with postnatal age in term and preterm infants.
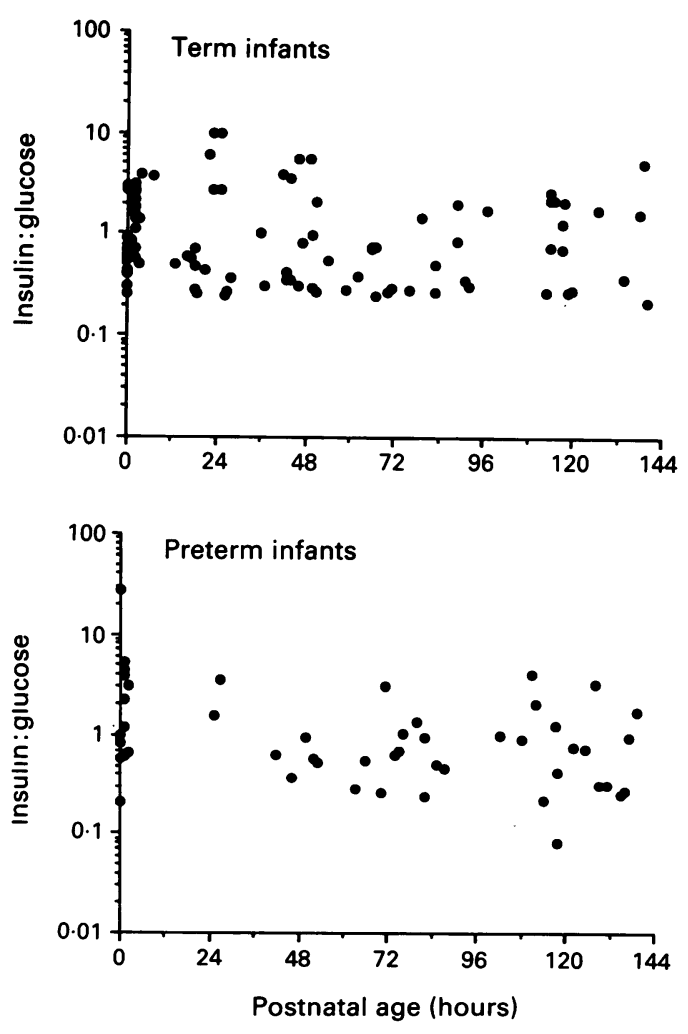

Figure 2 Relationship of plasma insulin:blood glucose ratio with postnatal age in term and preterm infants. 
blood glucose and cord plasma immunoreactive insulin concentrations. For all the infants, cord plasma immunoreactive insulin concentrations were not related to gestational age, birth weight, nor to birthweight SD score.

After birth, plasma immunoreactive insulin concentrations and insulin:glucose ratio did not vary with postnatal age in either term or preterm infants (fig 1 and 2). However, both blood glucose and plasma immunoreactive insulin concentrations were significantly higher in preterm than term infants (glucose, $\mathrm{p}<0.05$; insulin $\mathrm{p}<0.01$ ).

In children, there was a positive relationship between plasma immunoreactive insulin concentration and age $(r=056, \mathrm{p}<0.001)$. When the data from infants and children were compared, the ranges of plasma immunoreactive insulin concentrations were greater for both groups of infants than for children (table). Although preterm infants had significantly lower blood glucose concentrations than children, they had significantly higher plasma immunoreactive insulin concentrations $(p<0.01, p<0.001$, respectively). Similarly, term infants also had lower blood glucose concentrations, but higher plasma immunoreactive insulin concentrations, than children $(\mathrm{p}<0.001, \mathrm{p}<0.001$, respectively).

The insulin/glucose relationships for each group are shown in fig 3, which demonstrates that for children, insulin could not be detected in plasma when blood glucose concentrations were below $4.5 \mathrm{mmol} / \mathrm{l}$. However, in both groups of infants, insulin was detectable even when blood glucose concentrations were as low as $2 \cdot 0-2.5 \mathrm{mmol} / \mathrm{l}$. In preterm infants, both cord plasma immunoreactive insulin and postnatal plasma immunoreactive insulin concentrations were related to the respective blood glucose concentrations $(r=0.80, \mathrm{p}<0.01 ; r=0.44, \mathrm{p}<0.01$; respectively). In term infants, the cord blood glucose and plasma immunoreactive insulin concentrations were not related, but, after birth, there was a significant relationship between plasma immunoreactive insulin and blood glucose concentrations $(r=0.31 ; \mathrm{p}<0.01)$. This was also true in children, for whom there was a positive relationship between blood glucose and plasma immunoreactive insulin concentrations $r=0.58, \mathrm{p}<0.001$ ).

The independent associations of gestational age and blood glucose concentration, respectively, with plasma immunoreactive insulin concentration were examined using multiple regression analysis. Both gestational age and blood glucose concentration were independently correlated with plasma immunoreactive insulin concentration (for gestational age, $\mathrm{B}=-0.32$, SE $\mathrm{B}=0.04, \mathrm{p}<0.001$; for blood glucose, $B=0 \cdot 14, S E B=0 \cdot 03, p<0 \cdot 001)$. When the children were added into the analysis, multiple regression analysis, demonstrated that both maturity (that is whether being a preterm infant, a term infant, or a child) and blood glucose concentration were independently correlated with plasma immunoreactive insulin concentration (for maturity, $B=-0 \cdot 30$,
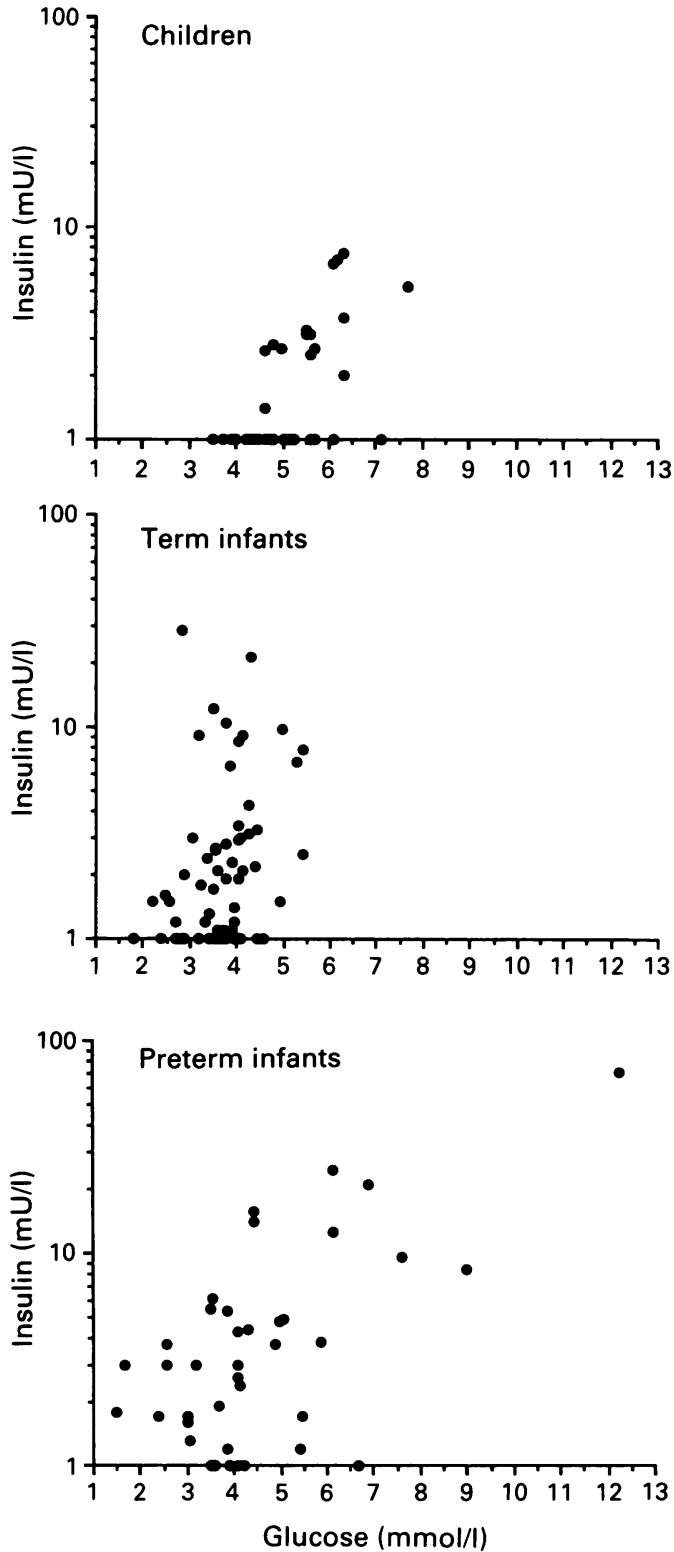

Figure 3 Insulin/glucose relationships in children and term and preterm infants.

SE $B=0.05, p<0.001$; for blood glucose, $\mathrm{B}=0 \cdot 14$, SE $\mathrm{B}=0 \cdot 03, \mathrm{p}<0 \cdot 001$ ).

Finally, the relationship between plasma NEFA and plasma immunoreactive insulin concentrations were examined. Plasma NEFA concentrations were low in both groups of infants, both at birth and after birth, and preterm infants, in particular, had very low NEFA concentrations. In term infants only, the cord plasma NEFA concentrations were negatively related to plasma immunoreactive insulin levels $(r=-0.42, \mathrm{p}<0.01)$. However, after controlling for cord blood glucose concentration, using multiple regression analysis, there was no relationship between cord plasma NEFA and immunoreactive insulin concentrations.

The relationships between plasma NEFA and immunoreactive insulin concentrations, in postnatal samples, are shown in fig 4 . After birth, for both term and preterm infants, there were negative relationships between plasma NEFA and immunoreactive insulin concen- 

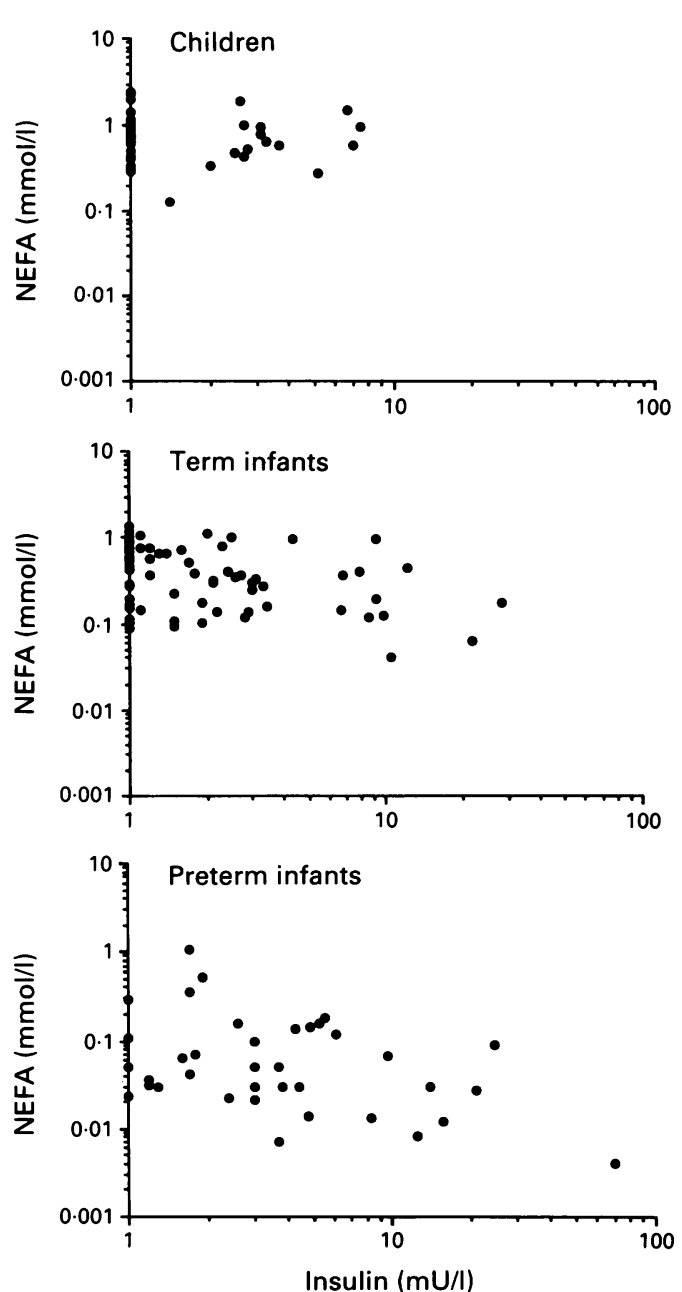

Figure 4 Relationship between plasma NEFA and insulin concentrations in children and term and preterm infants.

trations $r=-0 \cdot 30, \mathrm{p}<0 \cdot 01 ; r=-0 \cdot 29, \mathrm{p}=0 \cdot 038$ ). However, fig 4 demonstrates that the variation in NEFA concentration between infants in both groups, when compared with the median concentrations, was wide. Therefore, any variation in NEFA concentration associated with changing insulin concentrations was small in comparison to the variation between subjects. In addition, multiple regression analysis demonstrated that when gestational age and blood glucose concentration were added to the analysis, as independent variables, and plasma NEFA concentration was the dependent variable, there was no residual correlation between plasma immunoreactive insulin and NEFA concentrations (for gestational age $v \mathrm{NEFA}, \mathrm{B}=0.06, \mathrm{SE} \mathrm{B}=0.004, \mathrm{p}<0.001$; for glucose $v$ NEFA, $B=0 \cdot 12, \quad S E B=0.02$, $\mathrm{p}<0.001)$. Similarly, when the data from the children were added to the analysis, maturity and blood glucose concentration only were independently related to plasma NEFA concentration (for maturity $v$ NEFA, $B=0 \cdot 60, S E$ $\mathrm{B}=0.04, \mathrm{p}<0.001$; for glucose $v$ NEFA, $B=0 \cdot 12$, SE $B=0 \cdot 02, p<0 \cdot 001$ ).

Figure 5 demonstrates the relationship between blood glucose and plasma NEFA concentrations for term infants. The three infants (denoted*) who differed from the others in having low plasma NEFA concentrations at low blood glucose concentrations,

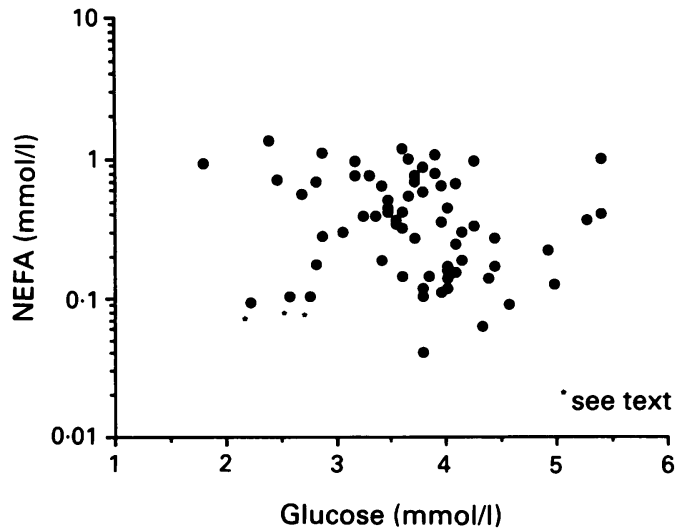

Figure 5 Relationship between plasma NEFA and blood glucose concentrations for term infants

had plasma immunoreactive insulin concentrations of $1.5,1.5$, and $<1.0 \mathrm{mU} / 1$, respectively.

\section{Discussion}

This study is the first to demonstrate the interrelationships between blood glucose and intermediary metabolite concentrations and plasma immunoreactive insulin concentrations in healthy AGA infants, and to compare these relationships with those of older children. We have already demonstrated that differences exist between term and preterm infants in the patterns of metabolic adaptation after birth, and between preterm infants, term infants, and children in the lipolytic and ketogenic response to low blood glucose concentrations. ${ }^{12}$ This report gives further information regarding the role of insulin in these changes, and compares the relationships found in neonates with those in children.

Although there was a positive relationship between blood glucose and plasma immunoreactive insulin concentrations in healthy term and preterm infants, this relationship differed from that found in older children. Basal insulin secretion (that is at low blood glucose concentrations) appeared to be higher for infants than for children, and the variation of plasma insulin concentrations in infants was greater than in children. Plasma insulin concentrations did not vary with increasing postnatal age, even after controlling for blood glucose concentration, but there was a spectrum of decreasing plasma insulin concentrations, from preterm infants to term infants to children. By controlling for blood glucose concentrations using multiple regression analysis, we have demonstrated that this was not simply the result of higher blood glucose concentrations. Therefore gestational immaturity itself appears to be associated with higher plasma immunoreactive insulin concentrations. However, after the neonatal period, during childhood, fasting insulin concentrations increased with age.

It has been demonstrated that term infants had high blood ketone body concentrations when blood glucose concentrations were low. ${ }^{12}$ It is possible that ketone bodies were utilised as alternative fuels by the neonatal brain, and thus prevented the neuroendocrine 
response to hypoglycaemia, which would otherwise have resulted in suppression of insulin secretion. This explanation would not apply to preterm infants, however, who had low blood ketone body concentrations even when blood glucose concentrations were low.

The present study did not examine the effects of increased plasma insulin concentrations on glucose metabolism. It is possible that, first, high basal insulin secretion may be the explanation of the low blood glucose concentrations found in a number of neonatal subjects. Alternatively, insulin resistance may exist in the neonatal period, in that insulin may not regulate hepatic glucose production or peripheral glucose uptake, and that other mechanisms, for example delayed induction of gluconeogenic enzymes, are responsible for the low blood glucose concentrations. Glucose/insulin clamp studies and glucose turnover studies in similar groups of healthy infants would be required to investigate these alternatives, by demonstrating whether insulin resistance existed and whether plasma insulin concentrations correlated with glucose production rates.

The present study yields some information on the role of insulin in fatty acid metabolism in neonatal subjects. Although there was a weak relationship between plasma insulin and NEFA concentrations, there was marked intersubject variability, and it appears that the effects of maturity and of circulating blood glucose concentrations were more important. In addition, those term infants who appeared to have a reduced lipolytic response to low blood glucose concentrations did not have high plasma insulin concentrations. Therefore the low NEFA concentrations and the impaired lipolytic response, found in preterm infants and some term infants, ${ }^{12}$ are more likely to be the result of other mechanisms, such as reduced adipose tissue stores, reduced counterregulatory hormone response, or immature enzyme systems. Fatty acid and ketone body turnover studies, with stable isotopically labelled substrates, would provide further information.

These data are useful in the interpretation of studies of hypoglycaemic and hyperglycaemic neonates. Previous studies have suggested that 'hyperinsulinism' may exist in asphyxiated and small for gestational age infants, in that plasma insulin concentrations were high when blood glucose concentrations were low. ${ }^{14-18}$ However, the present study has demonstrated that the same situation pertains in healthy, asymptomatic AGA infants. We suggest that the diagnosis of neonatal hyperinsulinism should be made on clinical grounds, namely a high intravenous glucose requirement to maintain normoglycaemia, and, in some cases, such as the islet cell dysregulation syndrome, the presence of macrosomy. ${ }^{20}$ This study demonstrates that it may be misleading to use biochemical data from older subjects as reference values for neonates, and, whenever possible, reference data from healthy neonates should be used.

It is possible that, in the radioimmunoassay for insulin, there has been cross reaction with substances that do not have the biological activity of insulin. Most insulin assays in current use show cross reactivity with proinsulin and proinsulin split products, ${ }^{21}$ and these molecules have low insulin-like biological activity. ${ }^{22}$ In normal adults this amounts to less than $10 \%$ of the immunoreactivity, but in normal neonatal subjects, at birth, the proportion of immunoreactivity accounted for by proinsulin is variable and ranges from 29 to $100 \% .^{23}$ In addition, it has been shown that, in infants of diabetic mothers, proinsulin concentrations were negatively correlated with gestational age. ${ }^{23}$ It has been suggested that the hypersecretion of proinsulin, by neonates, is the result of poorly developed storage granules, so that proinsulin is not fully converted to insulin and $C$ peptide. In the present study, there was a negative correlation between total immunoreactive insulin concentrations and gestational age, but we were unable to assess whether this relationship was entirely due to variation in proinsulin concentrations. There have been no studies, to date, which have measured the various components of immunoreactive insulin beyond the time of delivery in a group of normal subjects. It is possible that the relative proportions of proinsulin, insulin, and other molecules vary with postnatal age. Therefore, it may be inappropriate to compare data from cord blood of normal infants with those of abnormal infants, such as those who are hyperinsulinaemic. ${ }^{24}$ It is important that further studies are performed, using a specific assay, to measure the separate contributions of these molecules to the immunoreactive insulin concentrations in normal term and preterm infants after birth. Such studies will allow the quantification of the concentrations of biologically active insulin and its role in neonatal metabolic control.

Finally, the roles of enteral feeding and the enteroinsular axis in the control of pancreatic insulin secretion should be considered. It has been demonstrated that the gut hormone secretory responses to feeding develop with gestational and postnatal age, and these hormones, in turn, may influence insulin secretion. ${ }^{25} 26$ These relationships could not be investigated in the present study, but future studies of endocrine and metabolic interrelationships in the neonatal period should also examine the effects of feeding patterns and practices.

The group of children studied was heterogeneous in terms of age, and insulin/glucose relationships varied, even within the group. In addition, the present study was unable to investigate the timing of the emergence of the appropriate pancreatic secretory response, as infants older than the first postnatal week, but younger than the second postnatal month, have not been studied. Similarly, the development of the appropriate pancreatic responses in preterm infants are still poorly understood. Therefore, it would be informative to study homogeneous groups of infants and children, to extend the age of study beyond the first postnatal week, and to study, longitudinally, 
preterm infants. For these studies, as outlined above, a highly specific assay should be used to measure, separately, the concentrations of proinsulin, proinsulin split products, C peptide, and insulin.

In conclusion, many healthy neonates have high circulating immunoreactive insulin concentrations. However, it is possible that insulin does not have a major glucoregulatory role in the neonatal period, so that measurement of insulin concentrations alone is not clinically useful. This study demonstrates the importance of obtaining data from normal infants, before diagnosing abnormal metabolic and endocrine relationships in groups of infants with clinical disorders.

Dr Hawdon was supported by the Scientific and Research Committee of Newcastle Health Authority and by a Foundation for the Study of Infant Deaths training fellowship. We thank the parents who gave consent for the inclusion of their babies in the study. We acknowledge the assistance of Ms A McGann with metabolite assays and Ms L Ashworth with insulin assays.

1 Turner RC, Johnson PC. Suppression of insulin release by fish insulin induced hypoglycaemia. Lancet 1973;i: 1483-5.

2 Gerich JE. Glucose counterregulation and its impact on diabetes mellitus. Diabetes 1988;37:1606-17.

3 Cook DL, Taborsky GJ. $\beta$ cell function and insulin secretion. In: Rifkin H, Porte D, eds. Ellenberg and Rifkin's diabetes mellitus, theory and practice. New York: Elsevier, 1990.

4 Saua L, Sherwin RS, Hendler R, Felig P. Influence of continuous physiologic hyperinsulinaemia on glucose kinetics and counterregulatory hormones in normal and diabetic humans. $\mathcal{f}$ Clin Invest 1977;63:849-57.

5 McGarry JD, Fostser DW. Hormonal control of ketogenesis. Arch Intern Med 1977;137:495-501.

6 Falorni A, Fracassini F, Massi-Benedetti F, Massi S. Glucose metabolism and insulin secretion in the newborn infant. Diabetes 1974; 23:172-8.

7 Shelley HJ, Bassett JM, Milner RDG. Control of carbohydrate metabolism in the fetus and newborn. Br Med Bull 1975;31:37-43.

8 van Assche FA, Hoet J, Jack PMB. Endocrine pancreas of the pregnant mother, fetus and newborn. In: Beard RW, the pregnant mother, fetus and newborn. In: Beard RW, York: Marcel Decker, 1984:127-52.

9 Sperling MA, Grajwer LA, Leake R, Fisher DA. Role of glucagon in perinatal glucose homeostasis. Metabolism 1976;25(suppl):1385-6.

10 Cowett RM, Tenenbaum D. Hepatic response to insulin in the control of glucose kinetics in the neonatal lamb. Metabolism 1987;36:1021-6.

11 Stanley CA, Andy EK, Baker L, Delivoria-Papadopolous $M$. Metabolic fuel and hormone responses to fasting in newborn infants. Pediatrics 1979;64:613-9.

12 Hawdon JM, Ward Platt MP, Aynsley-Green A. Patterns of metabolic adaptation for preterm and term infants in the first neonatal week. Arch Dis Child 1992;67:357-65.

13 Hawdon JM, Aynsley-Green A, Bartlett K, Ward Platt MP. The role of pancreatic insulin secretion in neonatal glucoregulation. II. Infants with disordered blood glucose homoeostasis. Arch Dis Child 1993;68:280-5.

14 Collins E, Leonard JE. Hyperinsulinism in asphyxiated and small-for-dates infants with hypoglycaemia. Lancet 1984;ii:311-7.

15 Klenka HM, Scager J. Hyperinsulinism in asphyxiated and small for dates infants with hypoglycaemia. Lancet 984;ii:97

16 Bhowmick SK, Lewandowski C. Prolonged hyperinsulinism and hypoglycaemia in an asphyxiated small for gestation infant. Case management and literature review. Clin Pediatr (Phila) 1989;28:575-8.

17 Collins JE, Leonard JV, Teale D, et al. Hyperinsulinaemic hypoglycaemia in small for dates babies. Arch Dis Child 1990;65:1118-20.

18 Hawdon JM, Weddell A, Aynsley-Green A, Ward Platt MP. Hormonal and metabolic response to hypoglycaemia in small for gestational age infants. Arch Dis Child 1993; 68:269-73.

19 Ward Platt MP, Tarbit MJ, Aynsley-Green A. The effects of anaesthesia and surgery on metabolic homeostasis in infancy and childhood. $₹$ Pediatr Surg 1990;25:472-8.

20 Aynsley-Green A, Polak JM, Gough MH, et al. Nesidioblastosis of the pancreas: defintion of the syndrome and the man of the pancreas. defintion of the syndrome and the managment of the severe neonatal hyperinsulina

21 Temple RC, Carrington CA, Lazio SD, et al. Insulin deficiency in non-insulin dependent diabetes. Lancet 1989;i: ciency

22 Peavy DC, Brunner MR, Duckworth WC, Hooker CS, Frank BH. Receptor binding and biological potency of several split form (conversion intermediates) of human proinsulin. Studies in cultured IM-9 lymphocytes and in vivo and in vitro in rats. $\mathcal{F}$ Biol Chem $1985 ; 260: 13989-94$.

23 Heding LG, Persson B, Stangenberg M. B-cell function in newborn infants of diabetic mothers. Diabetalogica 1980;19:427-32.

24 Aynsley-Green A, Jenkins P, Tronier B, Heding LG. Plasma proinsulin and C-peptide concentrations in children with hyperinsulinaemic hypoglycaemia. Acta Pediatr Scand 1984;73:359-63.

25 Aynsley-Green A. Metabolic and endocrine interrelationships in the human fetus and neonate. Am $\mathcal{F}$ Clin Nutr 1985;41 (suppl 2):399-417.

26 Lucas A. Ontogeny of the gut hormones and hormone related substances. Acta Paediatr Scand 1989;362(suppl): 80-7. 